\title{
Muscle strength capacities in elite football players after anterior cruciate ligament reconstruction
}

\author{
Andreja Milutinovic ${ }^{1}$, Nemanja CoPic ${ }^{1}$, Adam Petrovic ${ }^{1,2}$, \\ MILINKO DABOVIC ${ }^{3}$, DANICA JANICIJEVIC ${ }^{3,4,5 *}$ \\ ${ }^{1}$ Faculty of Sport, University "Union - Nikola Tesla“, Belgrade, Serbia. \\ ${ }^{2}$ Faculty of Physical Culture and Sports, Derzhavin Tambov State University, Tambov, Russia. \\ ${ }^{3}$ University of Belgrade, Faculty of Sport and Physical Education, The Research Centre, Belgrade, Serbia. \\ ${ }^{4}$ Research Academy of Human Biomechanics, The affiliated hospital of medical school of Ningbo University, \\ Ningbo University, Ningbo, China. \\ ${ }^{5}$ Faculty of Sports Science, Ningbo University, Ningbo, China.
}

\begin{abstract}
Purpose: The aim of this study was to explore the strength capacities of the injured and non-injured leg following a unilateral anterior cruciate ligament reconstruction. Methods: Eight elite soccer players (age $=25.5 \pm 3.9$ years; height $=1.83 \pm 0.04 \mathrm{~m}$; body mass $=$ $78.9 \pm 4.5 \mathrm{~kg}$ ) volunteered to participate in this study. Nine months after the anterior cruciate ligament reconstruction and just before initiating the full training process, the maximal peak torque of the quadriceps and hamstring muscles were measured at 60 and 180 degrees/second using isokinetic dynamometry. Obtained peak torques were used for calculating hamstring-to-quadriceps ratio, limb symmetry index, and to estimate maximal torque capacity using a two-velocity method (i.e., linear torque-velocity relationship modeled considering peak torque obtained at 60 and 180 degrees/second). Results: No differences were found between hamstring-to-quadriceps ratios of the injured and non-injured leg $(p=0.165)$, nor between limb symmetry index of the quadriceps and hamstring muscles $(p=0.985)$, regardless of the angular velocity applied during tests. The two-point method revealed significant differences between quadriceps and hamstring muscle groups both in the injured and non-injured leg $(p<0.001$; maximal estimated torque was higher for quadriceps compared to hamstring muscles), while the differences between same muscle groups of the different legs were not significant. Conclusions: Collectively, these findings indicate that 9 months were enough for reaching a certain strength level of the injured leg that permits a safe return to play.
\end{abstract}

Key words: isokinetic dynamometry, limb symmetry index, hamstring-to-quadriceps ratio, two-point method

\section{Introduction}

A number of studies have indicated a high incidence of the anterior cruciate ligament (ACL) injury in various sports [9], [29], [39], with the soccer being the one with the highest ACL incidence. This painful injury has an important economic consequence in professional soccer and it is accompanied with an enormous pressure for a quick return to play [2]. However, the rate of early return to play is not as high as it could be expected due to the prolonged rehabilitation period that follows usually necessary surgical treatment [11], [39]. Although the return to play rate within a year following ACL reconstruction is very high, only twothird of players are competing at the same level 3 years later [36]. Consequently, the return to play after ACL reconstruction has become a major focus in the related research literature [2], [10], [26]. The primary goal of ACL reconstruction post-operative process is quadriceps and hamstring strength increment [3]. The quadriceps is normally considered to play an important role in jumping and ball kicking, while the hamstrings control running activities and stabilize the knees during

\footnotetext{
* Corresponding author: Danica Janicijevic. University of Belgrade, Faculty of Sport and Physical Education, The Research Centre, Belgrade, Blagoja Parovica 156, Serbia. Phone: +381641600474, e-mail: jan.danica@gmail.com

Received: March 15th, 2020

Accepted for publication: April 1st, 2021
} 
turns or tackles [14]. Moreover, it seems that the knee flexors' contribution to joint stability becomes increasingly important as the limb velocity increases [17]. The rehabilitation progress is traditionally followed-up by implementing isokinetic testing protocols and its outcomes are considered an important factor when deciding about returning to play [23], [31]. However, there is still no explicit recommendation regarding when is the best time to return to participate, especially if the sport activity that someone is returning to is full of pivoting activities such in soccer [30].

The level of maximal strength capacities of the hamstring and quadriceps muscles is one of the crucial factors that should be considered prior returning to play [2]. Specifically, several studies have reported that the ratio between hamstring and quadriceps strength levels (i.e., $\mathrm{H} / \mathrm{Q}$ ratio) of the injured leg should reach similar values as in non-injured leg [23], [38]. The H/Q ratio have been used for decades for monitoring knee joint stability, and it has been also considered as an important tool for evaluating lower extremity injury risk and rehabilitation process [34]. Although it is difficult to generalize, the normal $\mathrm{H} / \mathrm{Q}$ ratio is usually considered to be between 0.3 and 1.0 depending of the angular velocity used for its assessment, being the higher ratio frequently obtained at higher angular velocities [24], [28]. Another important index frequently considered for making decision about returning to play is the limb symmetry index (i.e., LSI) which received consistent attention from the strength and conditioning community over the last decades [5]. The LSI refers to the difference in the performance and mechanical outputs between two legs, while low LSI can affect jumping performance, ability to quickly change direction, provoke injuries [5]. Therefore, besides calculating H/Q ratios physicians and coaches usually also consider the differences in mechanical outputs of both injured and non-injured leg (i.e., LSI) when deciding of an appropriate time for their athletes to retake full training process [1], [13], [38].

Retaking full training participation imply performing sport specific movements with the maximal intended force/torque. Although the H/Q ratio and LSI present basic tools for deciding about returning to play, they do not reveal the maximal level of force/torque capacity. The maximal torque capacity is usually explored by performing isometric tests or using low angular velocities (e.g., 30 and $60 \%$ s) [21]. However, according to Zemach et al. [40] and Raj et al. [33], the employment of the isokinetic tests under low angular velocities can provoke pain in the patients who are still recovering from ACL injury. A potential solution can be the utilization of the two-velocity method that opens a possibility to estimate maximal torque capacity by modeling linear torque-velocity relationship considering torques obtained under 60 and $180^{\circ} / \mathrm{s}$. The two-velocity method has been previously validated in the study of Grbic et al. [15], who reported its high validity during the isokinetic knee extension test, and in the study of Janicijevic et al. [21], who demonstrated its high validity during the isokinetic knee extension and flexion tests. Although, the two-velocity method have shown to be sensitive enough to discriminate between antagonistic muscle groups acting on the knee joint [22], to our knowledge, no previous study used two-velocity method to explore the differences in the maximal torque capacity between injured and noninjured leg.

Therefore, taking the importance of H/Q ratio and LSI for deciding about returning to play, and potential benefits of the two-velocity method (i.e., maximal torque capacity estimation) into consideration, we designed a study aiming to explore strength characteristics of the injured and non-injured leg just before starting a full training process. Specifically, we hypothesized that (1) the $\mathrm{H} / \mathrm{Q}$ ratio of the injured leg may not differ from the H/Q ratio obtained in non-injured leg, (2) LSI may not differ between quadriceps and hamstring muscles, and (3) the maximal torque capacity will not differ between injured and non-injured leg.

\section{Materials and methods}

\section{Participants}

Eight elite soccer players (age $=25.5 \pm 3.9$ years; height $=1.83 \pm 0.04 \mathrm{~m}$; body mass $=78.9 \pm 4.5 \mathrm{~kg}$ ) with more than 10 years of competition experience volonteered to participate in this study. All participants were competing in the highest league in different European countries and all of them suffered ACL injury 9 month prior the initiation of the study (inclusion criteria). The potential participants were excluded from the study if they presented any other musculoskeletal injury or suffered from any chronic disease that can negatively affect the results of present study or their health integrity. They were informed about isokinetic testing procedure and possible risks associated with isokinetic assessment. The study protocol was approved by the Faculty of sport and physical education University of Belgrade Review Board (Approval number: 484-2) and an informed consent in accordance with the principles of the Declaration of Helsinki was signed by all participants. 


\section{Study design}

The present study was designed to explore the strength characteristics of the elite soccer players who were suffering ACL injury. During a single session the PT of the quadriceps and hamstring muscles of both injured and non-injured leg were recorded at $60 \% \mathrm{~s}$ and $180 \%$ s. A total of 20 cycles $(2$ legs $\times 2$ velocities $\times 5$ consecutive cycles; 1 cycle $=$ hamstring concentric contraction + quadriceps concentric contraction) were performed during a testing session that was organized prior to full training process initiation and 9 months from ALC reconstruction occurrence. The order of testing different legs (injured and non-injured) and velocities (60 and $180^{\circ} / \mathrm{s}$ ) was randomized. All sessions were performed between 9 and 11 am under similar environmental conditions for all participants $\left(\sim 22{ }^{\circ} \mathrm{C}\right.$ and $\sim 60 \%$ humidity).

\section{Testing procedures}

All measurements were performed using a HUMAC-NORM isokinetic dynamometer (Computer Sports Medicine Inc., Stoughton, USA) 9 month after ACL reconstruction. Following an ACL reconstruction, all soccer players completed same 4 stage rehabilitation procedure. The main goal of the first stage that started right after surgery and lasted 6 weeks was to reduce swelling and increment local circulation through passive movements performed with a help of a physical therapist, followed by isometric contractions and range of motion increment. During the second stage, participants started walking independently, cycling on the stationary bicycle and started performing closed chaincontrolled exercises without additional resistance. The second stage lasted approximately until the 14th week. The third stage started with implementing closed chain-controlled exercises firstly with reduced range of motion, while participants started to run and jump when their injured leg reached $70 \%$ of the maximal isometric force of their non-injured leg [8]. The third stage finished with performing isokinetic exercises through full range of motion in the 20th week. The fourth stage lasted until the end of the 8 th month and consisted from introducing and performing specific soccer exercises firstly executing them with greater volume and lower intensity, and finally closing a fourth stage of postoperative process with soccer specific situations of great intensity which were performed using a ball and along with other soccer player (i.e., situation training).

At the end of the 8th month, participants' muscle qualities were assessed using isokinetic dynamometry. Prior to the testing initiation, each subject underwent a 5-minute warm-up on a stationary bicycle, followed by passive stretching exercises (15 seconds per muscle group) focused on the quadriceps, hamstrings, hip adductors, and calf muscles. After that, the participants were seated in the chair of the isokinetic dynamometer and were fixed to the apparatus using straps around pelvis, across chest and around thigh and malleoli. The axis of rotation of the dynamometer head was aligned with the lateral femoral epicondyle (see Fig. 1 for detailed experimental setup). Before each data collection set, a specific warm-up set consisting of 3 submaximal knee flexions/extensions separately for each leg at 60 and $180 \%$ s was performed. Following a specific warm-up and a 2-minute pause, the experimental trials we recorded. Specifically, participants were instructed to extend their lower leg from $90^{\circ}$ in the knee joint (starting position), until reaching full extension $\left(180^{\circ}\right.$ in the knee joint) and completing 5 cycles ( 5 flexions and 5 extensions). The recovery time between different sets was 2 minutes. The same experienced investigator supervised all the trials and was in charged for providing verbal encouragement. The professional activity of the tested soccer players was followed during a 2.5-year period in order to make sure that the decision for returning to play was correct and to assure that no re-injury occurred. The follow-up of the soccer players following a long rehabilitation process is important in order to refine our knowledge regarding the best criteria for allowing a safe return to play.

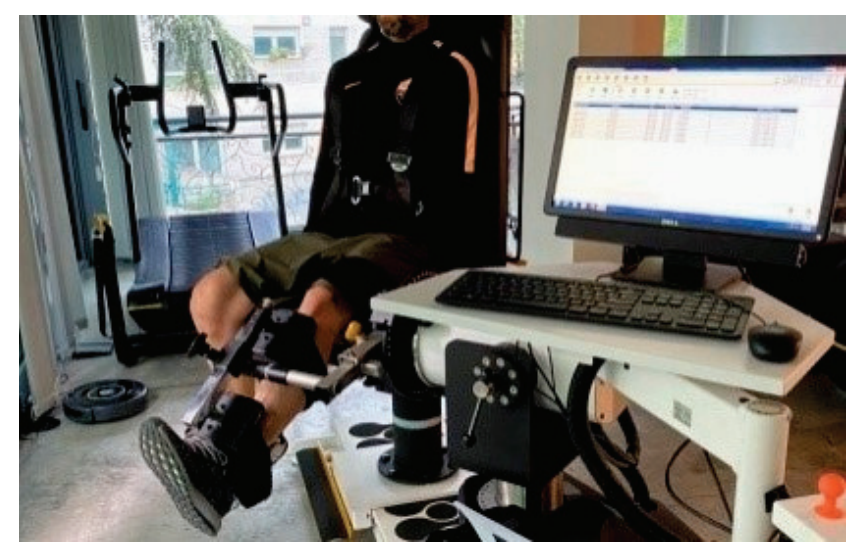

Fig. 1. Experimental setup

\section{Data analysis and analysis}

The HUMAC2009 V.10 software (Computer Sports Medicine Inc., Stoughton, USA) was used for data collection and data extraction. The maximal PT $(\mathrm{Nm})$ was used for calculating the H/Q ratios (for injured and non-injured leg) and limb symetry index (\%) (for hamstring and quadriceps muscle groups) using following equations: 


$$
\begin{gathered}
\text { HQ ratio }=\frac{\text { Hamstrings PT }}{\text { Quadriceps PT }}, \\
\mathrm{LSI}=\frac{\text { Injured PT }}{\text { Non }- \text { injured PT }} \times 100 \% .
\end{gathered}
$$

cally, four Friedman two-way analysis of the variance by ranks were used (1) exploring between-leg differences in peak torques obtained under different experimental conditions, (2) for exploring possible differences between $\mathrm{H} / \mathrm{Q}$ ratios obtained in injured and non-injured leg at $60^{\circ} / \mathrm{s}$ and $180^{\circ} / \mathrm{s}$, (3) for exploring

Table 1. Descriptive data of peak torque obtained under different velocities, legs and muscles

\begin{tabular}{|c|l|c|c|c|c|}
\hline \multicolumn{2}{|c|}{ Testing condition } & Injured & Non-injured & $P$-value & $\begin{array}{c}\text { Adjusted } \\
P \text {-value }\end{array}$ \\
\hline \multirow{2}{*}{$60^{\circ} / \mathrm{s}$} & Quadriceps & $253.0 \pm 218.3$ & $255.0 \pm 242.8$ & 0.683 & 1.000 \\
\cline { 2 - 6 } & Hamstrings & $132.0 \pm 125.0$ & $135.5 \pm 122.5$ & 0.919 & 1.000 \\
\hline \multirow{2}{*}{$180^{\circ} / \mathrm{s}$} & Quadriceps & $171.5 \pm 155.3$ & $176.5 \pm 171.0$ & 0.838 & 1.000 \\
\cline { 2 - 6 } & Hamstrings & $106.5 \pm 100.5$ & $108.0 \pm 94.0$ & 0.919 & 1.000 \\
\hline
\end{tabular}

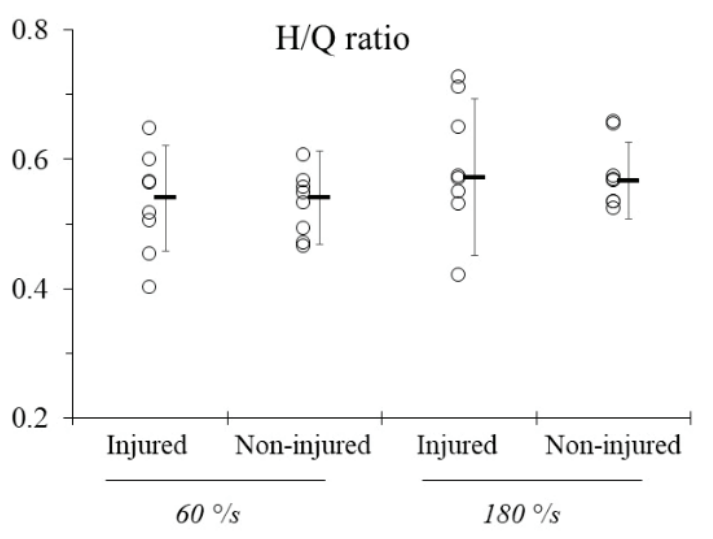

Fig. 2. The individual $\mathrm{H} / \mathrm{Q}$ ratios of the injured and non-injured leg recorded under $60^{\circ} / \mathrm{s}$ and $180^{\circ} / \mathrm{s}$, accompanied with median value (i.e., -) and error bars (i.e., interquartile range).

$$
\text { HQ ratio }=\frac{\text { Hamstrings PT }}{\text { Quadriceps PT }}
$$

Both the maximal PT $[\mathrm{Nm}]$ and the angular velocity $[\mathrm{rad} / \mathrm{s}]$ were used for modelling torque-velocity relationships using two-point method (i.e., considering two pairs of PT and angular velocities) and following linear regression model:

$$
T(\omega)=T_{0}-a \omega,
$$

where $T_{0}$ represents the torque-intercept (i.e., estimated theoretical PT), $a$ is the slope that corresponds to $T_{0} / \omega_{0}$, while $\omega$ is the angular velocity.

\section{Statistical analysis}

Descriptive data are presented through median and interquartile range values, while normality of the data was explored using Kolmogorov-Smirnov test. Since the data deviated from normal distribution, and relatively low number of soccer players participated in this study, we used non-parametric statistic. Specifi-

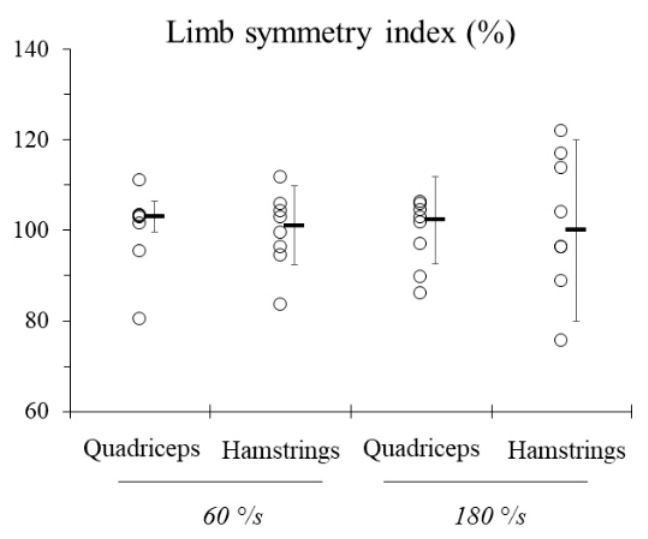

Fig. 3. The individual limb symmetry indexes of the quadriceps and hamstrings muscles recorded under $60 \%$ and $180 \%$, accompanied with median value (i.e., - ) and error bars (i.e., interquartile range). LSI $=\frac{\text { Injured PT }}{\text { Non }- \text { injured PT }} \times 100 \%$

possible differences between LSI obtained between legs for quadriceps and hamstring muscles at $60 \% \mathrm{~s}$ and $180 \% \mathrm{~s}$, and (4) for exploring possible differences between maximal torque capacities obtained modeling two-velocity method. $P$-values were adjusted using the Bonferroni corrections for multiple comparisons. Statistical analyses were performed using the software package SPSS (IBM SPSS version 22.0, Chicago, IL, USA). Statistical significance was set at an alpha level of 0.05 .

\section{Results}

A Kolmogorov-Smirnov test indicated that the PT recorded in our study do not follow a normal distribution $(p<0.05)$. The descriptive data of the PT 
obtained under different experimental conditions are depicted in Table 1. Regardless of the angular velocity used for recording PT, no differences were found between $\mathrm{H} / \mathrm{Q}$ ratios of the injured and non-injured leg $\left(\chi^{2}=5.1, p=0.165\right.$; Fig. 2$)$, nor between LSI (\%) of the quadriceps and hamstring muscles $\left(\chi^{2}=0.2\right.$, $p=0.985$; Fig. 3). These results indicate that just before initiation of the full training process the H/Q ratio of the injured leg was similar to the H/Q index of the non-injured leg, while the LSI were similar for the quadriceps and hamstring muscles under $60 \% \mathrm{~s}$ as well as under $180^{\circ} / \mathrm{s}$.

Data are presented as median \pm first interquartile range. No significant differences were obtained between injured and non-injured leg when same testing conditions were compared. $P \leq 0.05$.

Significant differences were detected between maximal torque capacity $(p<0.001)$ obtained for the quadriceps and hamstring muscles of the injured and noninjured leg. Pairwise comparisons showed that the values of maximal torque capacity were higher for quadriceps muscle of the non-injured leg in comparison with
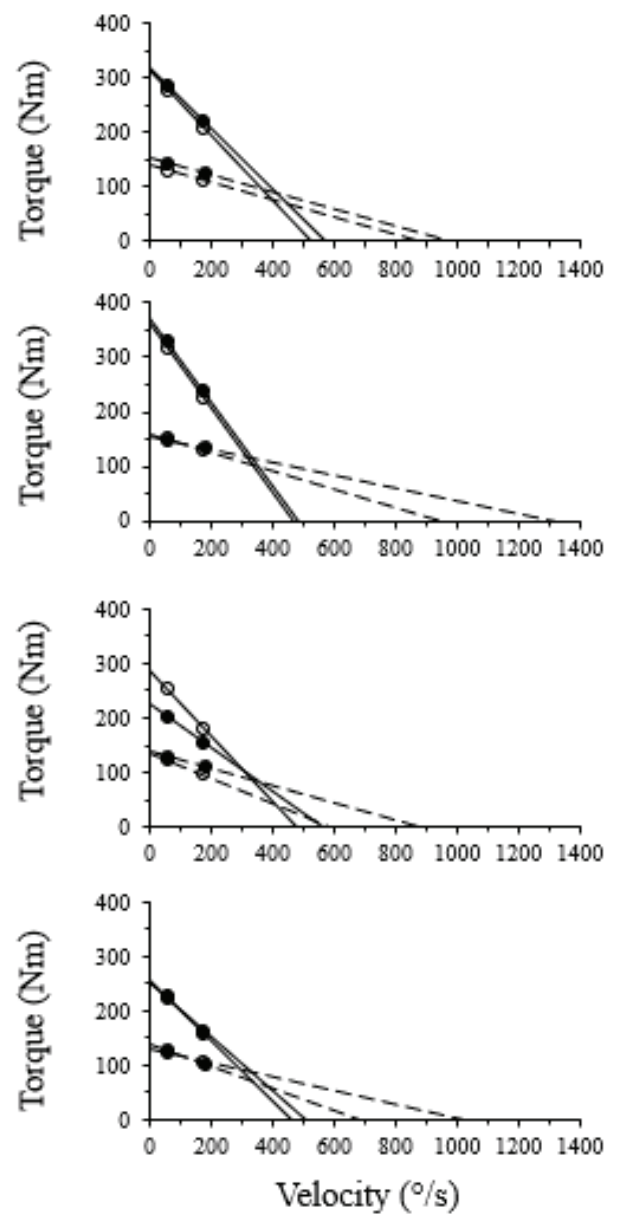

the hamstring muscles of the injured leg $(p=0.022)$, and for quadriceps muscles of the injured leg in comparison with hamstring muscles of the both injured $(p=0.001)$ and non-injured leg $(p=0.006)$, while in the rest of the comparisons no differences were found. The individual F-V relationships are depicted in the Fig. 4.

\section{Discussion}

This study was designed to explore strength capacities of the injured and non-injured leg just before starting a full training process. The main findings of the study were that (1) H/Q ratio did not differ between injured and non-injured leg, (2) LSI obtained for the quadriceps and hamstring muscles did not differed neither under $60 \%$ s nor under $180 \%$, and (3) maximal estimated PT were higher for the quadriceps muscles compared to hamstring muscles, while no differences were found between same muscle group when injured
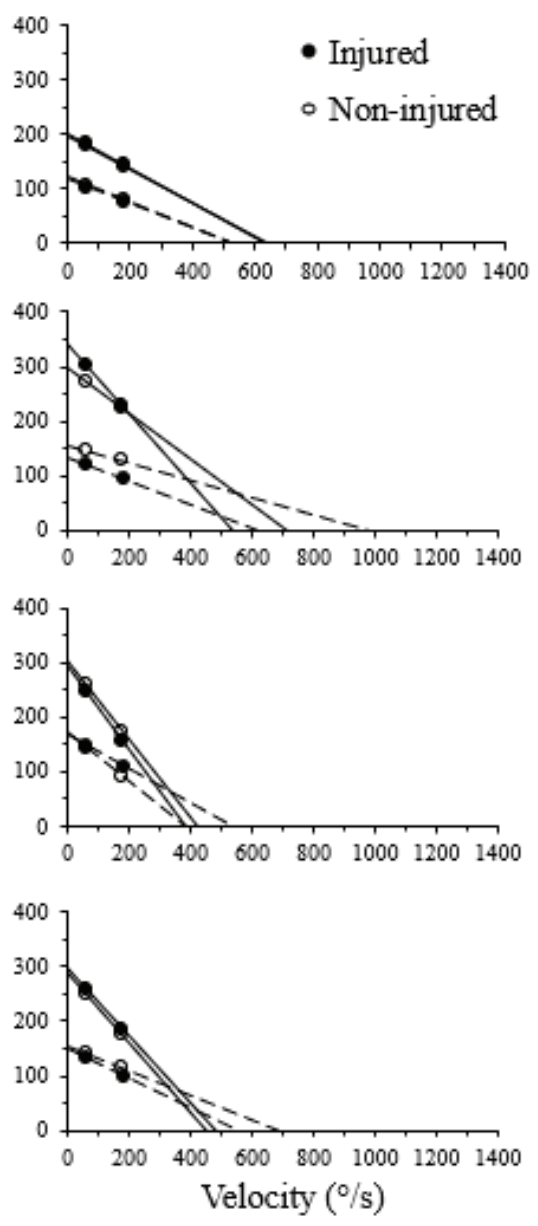

Fig. 4. Individual torque-velocity relationships modelled using two-point method and linear regression model.

The empty circles represent non-injured leg, while full circles injured one.

Full and dashed lines represent quadriceps and hamstring muscles, respectively 
and non-injured leg were compared. These findings indicate that the strength capacities of the injured leg reached values similar to those of the non-injured leg 9 months after the ACL reconstruction.

Confirming our first hypothesis, the $\mathrm{H} / \mathrm{Q}$ ratio did not differ between injured and non-injured leg, which is considered as a good quantitative sign for allowing the return to playing. The H/Q ratios obtained in our study are in line with the optimal range of $\mathrm{H} / \mathrm{Q}$ ratios proposed by other authors [24], [25]. However, the median $\mathrm{H} / \mathrm{Q}$ ratio obtained under $60 \%$ s (i.e., 0.54 ) is somewhat lower than reported in the studies of Kadija et al. [24] and Hole at al. [20] (i.e., 0.67), which can be explained by distinct relative time point for scheduling a testing sessions (i.e., 9 months after ACL reconstruction in our study compared to 4 months posterior to the ACL reconstruction in the study of Kadija et al. [24] and prior ACL reconstruction in the study of Hole at al. [20]). In accordance with other studies, we observed that the increment in the angular velocity in our study was accompanied with the increment of the H/Q ratio [24], [20], [35]. Regarding the LSI, it never differed between compared conditions (i.e., hamstring LSI $[60 \%$ s] vs. hamstring LSI $[180 \%$ s] vs. quadriceps LSI $[60 \%$ s] vs. quadriceps LSI $[180 \%$ ] $)$, although in few cases, it went beyond usually recommended range of asymmetries (i.e., $<20 \%$ ) [4]. Even though in minority, these augmented differences (i.e., up to $25 \%$ ) were detected for the hamstring muscles at $180 \%$, which is in line with the previous studies [16], [19]. Generally, slower recuperation of the hamstring, compared to quadriceps muscles, especially pronounced during higher shortening velocities, can be the possible reason of obtaining these augmented differences [12], [18], [32]. And, although some studies indicated that LSI can overestimate knee function [31], [37], the isokinetic dynamometry is still a recommended as a sensitive for exploring strength differences between injured and non-injured leg [31].

Arthrogenic muscle inhibition, loss of mechanoreceptors, pain and swelling are some of the reasons of significant drop in the thigh muscle strength after ACL injury [6]. Rehabilitation process includes progressive strength training, which is usually prescribed with respect to the maximal strength capacities [6]. Apart from this, reporting maximal isometric and peak torques have been recommended as a useful and necessary tool for comprehensive post-operative muscle assessment [27]. Since it is not recommendable to actually measure maximal strength capacities until full range of motion and certain level of muscle mass are gained, in our study, we used two-velocity method for estimating maximal PT. No significant differences were obtained in the maximal PT between injured and non-injured leg, which indicates that 9 months were enough for the injured leg to reach the maximal PT level of the non-injured one. This finding is especially important because a relative PT deficit of the injured leg is frequently higher than $30-40 \%$ at the early stages of the rehabilitation [24], [28]. Although, to our knowledge, no study used two-velocity method to estimate PT in the participants recovering from ACL injury, Janicijevic et al. [22] demonstrated that the two-velocity method was sensitive enough to discriminate between maximal strength capacities of the antagonistic muscle groups in healthy active and sedentary males. Specifically, they found that higher force values were obtained for the quadriceps in comparison to the hamstring muscles, which is in line with the findings of our study. Therefore, this study confirms the sensitivity of the two-velocity method to discriminate between PT values obtained in antagonistic muscle groups, but at the same time opens a possibility to explore both H/Q ratio and LSI in fatigue-free and quick manner.

A few limitations should be acknowledged and considered for future studies. First, the low number of the participants was a consequence of the rigorous criteria for someone to be included in this study (elite athletes with a minimum of 10 years of soccer experience and ACL reconstruction). Secondly, we could not systematically follow-up the strength progress of all soccer players during postoperative process due to the different places of residence and centers in which our participants underwent rehabilitation procedure. Thirdly, we failed to include a control group (i.e., elite soccer players without ACL injury) due to the dense schedules of the professional soccer players and their lack of interest to participate as a part of the control group. And finally, it would be interesting to compare the results of the isokinetic test with the results of the more functional tests (i.e., jumps, lunges, etc.).

\section{Conclusions}

An isokinetic dynamometry has been widely used as an objective parameter for taking decision about returning to play following an ACL reconstruction [7]. Our study revealed that 9 months after ACL reconstruction, differences between injured and non-injured obtained during isokinetic tests (i.e., at 60 and $180^{\circ}$ s) were minimal. Specifically, no differences were found in the magnitudes of the $\mathrm{H} / \mathrm{Q}$ ratios between legs, nor between LSI for the quadriceps and hamstring mus- 
cles. The two-velocity method was sensitive enough to reveal differences in the maximal estimated PT between quadriceps and hamstring muscles, while no differences were found between same muscle group of the injured and non-injured leg. Collectively, these findings indicate that the 9 months were enough for reaching certain level of recovery that permits return to playing. The information obtained in this study can be considered valuable, given that none of the soccer players had recurrent ACL injuries for an average of 2.5 years from the start of the full training and competition process.

\section{Acknowledgements}

The research is a part of the project III47015 and was supported by the grants 451-03-68/2020-14/200015, 451-03-68/2020-14/ 200021 from the Ministry of education, science, and technological development of Republic of Serbia.

\section{References}

[1] Ageberg E., Roos H.P., Silbernagel K.G., ThomeÉ R., Roos E.M., Knee extension and flexion muscle power after anterior cruciate ligament reconstruction with patellar tendon graft or hamstring tendons graft: A cross-sectional comparison 3 years post surgery, Knee Surgery, Sports Traumatology, Arthroscopy, 2009, 17, 162-169, DOI: 10.1007/s00167-008-0645-4.

[2] Arliani G.G., Pereira V.L., Leão R.G., Lara P.S., EJNISMAN B., COHEN M., Treatment of anterior cruciate ligament injuries in professional soccer players by orthopedic surgeons, Revista Brasileira de Ortopedia, 2019, 54, 703-708, DOI: $10.1055 / \mathrm{s}-0039-1697017$.

[3] Arundale A.J.H., Silvers-Granelli H.J., SNYDER-Mackler L., Career length and injury incidence after anterior cruciate ligament reconstruction in major league soccer players, Orthopaedic Journal of Sports Medicine, 2018, 6, 2325967117750825, DOI: $10.1177 / 2325967117750825$.

[4] BARBER-Westin S.D., Noyes F.R., Factors used to determine return to unrestricted sports activities after anterior cruciate ligament reconstruction, Arthroscopy - Journal of Arthroscopic and Related Surgery, 2011, 27, 1697-1705, DOI: 10.1016/ j.arthro.2011.09.009.

[5] Bishop C., Read P., Chavda S., Turner A., Asymmetries of the lower limb: The calculation conundrum in strength training and conditioning, Strength \& Conditioning Journal, 2016, 38, 27-32, DOI: 10.1519/SSC.0000000000000264.

[6] Buckthorpe M., La Rosa G., Villa F. Della, Restoring knee extensor strength after cruciate ligament reconstruction: A clinical commentary, International Journal of Sports Physical Therapy, 2019, 14, 159-172, DOI: 10.26603/ijspt20190159.

[7] Cvjetkovic D.D., Bijeljac S., Palija S., Talic G., Radulovic T.N., Kosanovic M.G., Manojlovic S., Isokinetic testing in evaluation rehabilitation outcome after $A C L$ reconstruction, Medical archives (Sarajevo, Bosnia and Herzegovina), 2015, 69, 21-23, DOI: 10.5455/ medarh.2015.69.21-23.
[8] Czamara A., Moments of muscular strength of knee joint extensors and flexors during physiotherapeutic procedures following anterior cruciate ligament reconstruction in males, Acta Bioeng. Biomech., 2008, 10, 37-44.

[9] Dodson C.C., Secrist E.S., Bhat S.B., Woods D.P., DeluCA P.F., Anterior cruciate ligament injuries in national football league athletes from 2010 to 2013: A descriptive epidemiology study, Orthopaedic Journal of Sports Medicine, 2016, 4 (3), 2325967116631949 , DOI: 10.1177/2325967116631949.

[10] Ebert J.R., Edwards P., Yi L., Joss B., Ackland T., CAREY-SMith R., Buelow J.U., HewitT B., Strength and functional symmetry is associated with post-operative rehabilitation in patients following anterior cruciate ligament reconstruction, Knee Surgery, Sports Traumatology, Arthroscopy, 2018, 26, 2353-2361, DOI: 10.1007/s00167-017-4712-6.

[11] Farber J., Harris J.D., Kolstad K., McCulloch P.C., Treatment of anterior cruciate ligament injuries by major league soccer team physicians, Orthopaedic Journal of Sports Medicine, 2014, 2, 1-7, DOI: 10.1177/2325967114559892.

[12] FaXON J.L., Sanni A.A., McCully K.K., Hamstrings and quadriceps muscles function in subjects with prior ACL reconstruction surgery, Journal of Functional Morphology and Kinesiology, 2018, 3, DOI: 10.3390/jfmk3040056.

[13] Fischer F., Fink C., Herbst E., Hoser C., Hepperger C., BlANK C., GFÖLLER P., Higher hamstring-to-quadriceps isokinetic strength ratio during the first post-operative months in patients with quadriceps tendon compared to hamstring tendon graft following ACL reconstruction, Knee Surgery, Sports Traumatology, Arthroscopy, 2018, 26, 418-425, DOI: 10.1007/s00167-017-4522-x.

[14] Fried T., Lloyd G.J., An overview of common soccer injuries: Management and prevention, Sports Medicine, 1992, 14, 269-275, DOI: 10.2165/00007256-199214040-00005.

[15] Grbic V., DJuric S., Knezevic O., Mirkov D., NedelJKovic A., JARIC S., A novel two-velocity method for elaborate isokinetic testing of knee extensors, International Journal of Sports Medicine, 2017, 38, 741-746, DOI: 10.1055/s-0043-113043.

[16] HÄGGLUND M., WALdÉN M., EKSTRAND J., Risk factors for lower extremity muscle injury in professional soccer: The UEFA injury study, American Journal of Sports Medicine, 2013, 41, 327-335, DOI: 10.1177/0363546512470634.

[17] Hagood S., Solomonow M., Baratta R., Zhou B.H., D'AmBrosia R., The effect of joint velocity on the contribution of the antagonist musculature to knee stiffness and laxity, The American Journal of Sports Medicine, 1990, 18, 182-187, DOI: $10.1177 / 036354659001800212$.

[18] HaRput G., Ozer H., BAltaCi G., Quadriceps muscle strength recovers faster than hamstring strength after ACL reconstruction with hamstring tendon autograft, Medicine and Science in Sports and Exercise, 2017, 49, 955-956, DOI: 10.1249/01.mss.0000519606.58138.be.

[19] Holcomb W.R., Rubley M.D., Lee H.J., Guadagnoli M.A., Effect of hamstring-emphasized resistance training on hamstring:quadriceps strength ratios, Journal of Strength and Conditioning Research, 2017, 21, 41-47, DOI: 10.1519/ R-18795.1.

[20] Hole C.D., Hammond J., Smith G.H., Kumar A., SaXton J., Cochrane T., Dynamic control and conventional strength ratios of the quadriceps and hamstrings in subjects with anterior cruciate ligament deficiency, Ergonomics, 2000, 43, 1603-1609, DOI: 10.1080/001401300750004023.

[21] Janicijevic D., García-Ramos A., KnezeviC O.M., Mirkov D.M., Feasibility of the two-point method for as- 
sessing the force-velocity relationship during lower-body and upper-body isokinetic tests, Journal of Sports Sciences, 2019, 37, 2396-2402, DOI: 10.1080/02640414.2019.1636523.

[22] JaniciJevic D., Knezevic O.M., Garcia-Ramos A., Cvetic D., MIRKOV D.M., Isokinetic testing: Sensitivity of the force-velocity relationship assessed through the two-point method to discriminate between muscle groups and participants' physical activity levels, International Journal of Environmental Research and Public Health, 2020, 17, 8570, DOI: 10.3390/ijerph17228570.

[23] Kadija M., Knezević. O., Milovanovic D., Nedeljkovic A., MiRKov D., The effect of anterior cruciate ligament reconstruction on hamstring and quadriceps muscle function outcome ratios in male athletes, PubMed.. Srp. Arh. Celok. Lek., 2016, 144, 151-157.

[24] Kadija M., Knezevic O., Milovanovic D., Bumbasirevic M., MiRKOV D.M., Effect of isokinetic dynamometer velocity on muscle strength deficit in elite athletes after ACL reconstruction, Medicina dello Sport, 2010, 63 (4), 495-507.

[25] Kannus P., Peak torque and total work relationship in the thigh muscles after anterior cruciate ligament injury, Journal of Orthopaedic and Sports Physical Therapy, 1988, 10, 97-101, DOI: 10.2519/jospt.1988.10.3.97

[26] Kobayashi A., Higuchi H., Terauchi M., Kobayashi F., KIMURA M., TAKAGISHI K., Muscle performance after anterior cruciate ligament reconstruction, International Orthopaedics, 2004, 28, 48-51, DOI: 10.1007/s00264-003-0502-5.

[27] KrZEmińsKa K., CZAmara A., Diagnostic value of the hamstring to quadriceps ratio in monitoring of the effectiveness of supervised 6-month physiotherapy in males after Anterior Cruciate Ligament Reconstruction (ACLR), Acta Bioeng. Biomech., 2020, 22, 31-39.

[28] MAfFulli N., Isokinetic strength of the quadriceps and hamstrings and functional ability of anterior cruciate deficient knees in recreational athletes, British Journal of Sports Medicine, 1996, 30, 161-164, DOI: 10.1136/bjsm.30.2.161.

[29] Moses B., Orchard J., ORChard J., Systematic review: Annual incidence of ACL injury and surgery in various populations, Research in Sports Medicine, 2012, 20, 157-179, DOI: 10.1080/15438627.2012.680633.

[30] Myklebust G., BAHR R., Return to play guidelines after anterior cruciate ligament surgery, British Journal of Sports Medicine, 2005, 39, 127-131.

[31] Nagai T., Schilaty N.D., Laskowski E.R., Hewett T.E., Hop tests can result in higher limb symmetry index values than isoki- netic strength and leg press tests in patients following ACL reconstruction, Knee Surgery, Sports Traumatology, Arthroscopy, 2020, 28, 816-822, DOI: 10.1007/s00167-019-05513-3.

[32] Neeter C., Gustavsson A., ThomeÉ P., Augustsson J., ThomeÉ R., KARLSSON J., Development of a strength test battery for evaluating leg muscle power after anterior cruciate ligament injury and reconstruction, Knee Surgery, Sports Traumatology, Arthroscopy, 2006, 14, 571-580, DOI: 10.1007/ s00167-006-0040-y.

[33] RAJ I.S., Bird S.R., SHIELD A.J., Aging and the force - velocity relationship of muscles, Experimental Gerontology, 2010, 45, 81-90, DOI: 10.1016/j.exger.2009.10.013.

[34] Ruas C.V., Pinto R.S., Haff G.G., Lima C.D., Pinto M.D., BROwn L.E., Alternative methods of determining hamstringsto-quadriceps ratios: a comprehensive review, Sports Medicine - Open, 2019, 5, 11, DOI: 10.1186/s40798-019-0185-0.

[35] Struzik A., Siemienski A., Tadeusz B., PietraszewaKi B., Ratios of torques of antagonist muscle groups in female soccer players, Acta Bioeng. Biomech., 2018, 20, 153-158.

[36] WaldÉn M., HägGlund M., MagnuSSON H., EKSTRAND J., ACL injuries in men's professional football: A 15-year prospective study on time trends and return-to-play rates reveals only $65 \%$ of players still play at the top level 3 years after ACL rupture, British Journal of Sports Medicine, 2016, 50, 744-750, DOI: 10.1136/bjsports-2015-095952.

[37] Wellsandt E., Failla M.J., SNYDER-Mackler L., Limb symmetry indexes can overestimate knee function after anterior cruciate ligament injury, Journal of Orthopaedic and Sports Physical Therapy, 2017, 47, 334-338, DOI: 10.2519/ jospt.2017.7285

[38] Willigenburg N.W., McNally M.P., Hewett T.E., Quadriceps and hamstrings strength in athletes, Hamstring and quadriceps injuries in athletes, 2014, 15-28, DOI: 10.1007/ 978-1-4899-7510-2_2.

[39] Wrzesien Z., Truszczynska-BaszaK A., RzEPKA R., Lower extremity muscle strength, postural stability and functional movement screen in female basketball players after ACL reconstruction. Preliminary report, Acta Bioeng. Biomech., 2019, 21, 71-81.

[40] Zemach L., Almoznino S., BaraK Y., Dvir Z., Quadriceps insufficiency in patients with knee compromise: How many velocities should an isokinetic test protocol consist of?, Isokinetics and Exercise Science, 2009, 17, 129-133, DOI: 10.3233/IES-2009-0344. 